\title{
Optimal Control of the Parameters of the Production Line
}

\author{
Oleh Pihnastyi \\ National Technical University "Kharkiv Polytechnic Institute", Kharkov, Ukraine \\ *Corresponding Author: Oleh Pihnasty, National Technical University "Kharkiv Polytechnic Institute", \\ Kharkov, Ukraine

\begin{abstract}
The problem of optimal control of the parameters of the production flow line - stocks (work in process) and the rate of processing of objects of labour for a technological operation is considered. The article presents a mathematical formulation of the problem of controlling the parameters of a production line with restrictions on work in progress and the speed of machining parts for each technological operation. The control program is determined by the specified quality criteria. An example of the calculation of the optimal control for the production line parameters is presented.
\end{abstract}

Keywords: production line, PDE-model of production, balance equations, transition period, work in progress.

\section{Formulation of the Problem}

Production control theory is an intensively developing field of knowledge, the development of which is stimulated by practical needs [1,2]. Along with the traditional methods of designing control systems along precise lines [3-8] methods related to partial differential equations (PDE models) [9-12]. The main approaches to the design of production line control systems are based on program control and deviation control [13-15]. If, when moving along a technological route, the law of changing the parameters of objects of labor is known and known are external influences on the parameters of objects of labor, and the goal of managing the production process is achievable, then the law can be obtained control parameters of the production line for the period of the production program. If the perturbations are unknown, but can become measured at the time of the decision, then the control of the process parameters is formed as a function of their perturbations.

When designing production line control systems, an important step is to find an appropriate description model for the controlled process. The classical theory of optimal control $[16,17]$ widely used for dynamic systems design $[13,15,19,20]$, whose evolution is given by differential equations. The developed apparatus of the theory of optimal control can be successfully applied when building models of a controlled production process in the case when continuous models are used to describe them. [1]. The model of the controlled process should contain the parameters of the flow of products and the parameters of the state of the inter-operational backlogs in both stationary and transient modes, as they are key for production control [1]. In addition, the model should be able to provide a solution to the production task for a limited time using the specified computing resources.

\section{Analysis of ReCEnt DeVelopments ANd Publications}

In numerous publications devoted to the development and design of production line control systems, there are three main types of production systems models: mass service models (TQ-model), discrete event models (DES-model) [21,22] and continuous fluid models (Fluid -model) [2,23]. Well-proven models for describing quasistatic processes are not used to design transient control systems [1]. In the last decade, the design of control systems for production lines used models containing partial differential equations (PDE-model) $[9,12]$.

In the last decade, the design of control systems for production lines used models containing partial differential equations [24], combining the advantages of TQ-models, DES-models and Fluid-models, significantly expanded the capabilities of designing production line control systems. PDE-models being continuous can be successfully used in the description of stationary and transient modes of production of a production line and do not require a large expenditure of machine time [1]. 


\section{Formulation OF RESEARCH ObJECTIVES}

In modern economic conditions, the duration of the production cycle is a significant part of the product life cycle, as a result, the production lines for a significant part of the time operate in a transient unsteady mode.

Particular attention in the tasks of optimal control of modern production of products is occupied by transitional modes of operation of a production line with a length of up to a month, associated with the launch and increase of production volumes at the beginning of the product life cycle, and with the folding and stopping of production at the stage of completion of the life cycle [25,c.4589].

The next important issue is the study of optimal transition modes from one normative state of production line parameters to another, due to an increase or decrease in demand for manufactured products [1]. The present work is devoted to these areas of research.

\section{Main Material}

It is known [6,26-28] that in order to ensure continuous operation of the production, the value of the capacity of the production line must be related to the value of the interoperative reserve by a strictly defined dependence, equation of the continuity of the flow of objects of labor along the technological route [12]. In general, the control of interoperability backlog along the technological route is achieved through the use of a multithreaded line control model with reserve stock allocation [29] or by providing the required gradient of the rate of processing of objects of labor along the technological route (picture 1). The capacity control of a production line is controlled by gradually increasing the number of equipment units operating in parallel, directly through measures to change the processing modes of objects of labor with technological equipment [1]. We believe that the given: a) the sequence of technological operations and their technological parameters; b) the equipment necessary to perform the technological operation, the parameters of its work and the layout scheme; c) the properties of the object of labor and the laws of the transfer of technological resources to the objects of labor as a result of the impact of equipment. We introduce one-dimensional coordinate space $(t, S)$ [25,30,31]. Divide the coordinate axis $0 S$ into segments $\Delta S_{m} \in\left[S_{m-1}, S_{m}\left[\right.\right.$. Coordinate $S_{m-1} \quad$ (\$) and $S_{m}(\$)$ characterizes the beginning and ending $m-t h$ technological operation, $m=1 . . M$.At the same time, we assume that $S_{0}=0 \quad(\$), S_{M}=S_{d}(\$)$, where $S_{d}(\$)$ - production cost. We also believe that the price of the costs associated with the control for each technological operation is known, is different for each technological operation and depends on the time of day. The flow parameters of the production line model in the two-step description are interoperative backlogs characterized by density $[\chi]_{0}(t, S)$ , and the rate of movement of objects of labor $[\chi]_{1}\left(t, S_{d}\right)$ on the technological route [32]. We believe that the discrete control function with a sufficient degree of accuracy can be approximated by a continuous function

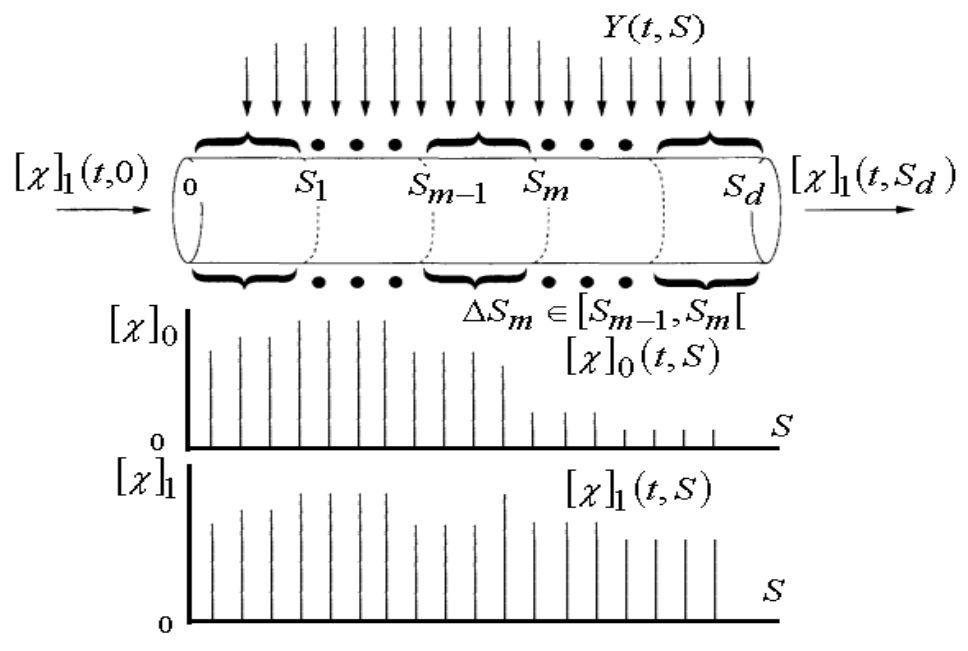

Fig1. Control model for distributed flow line parameters 


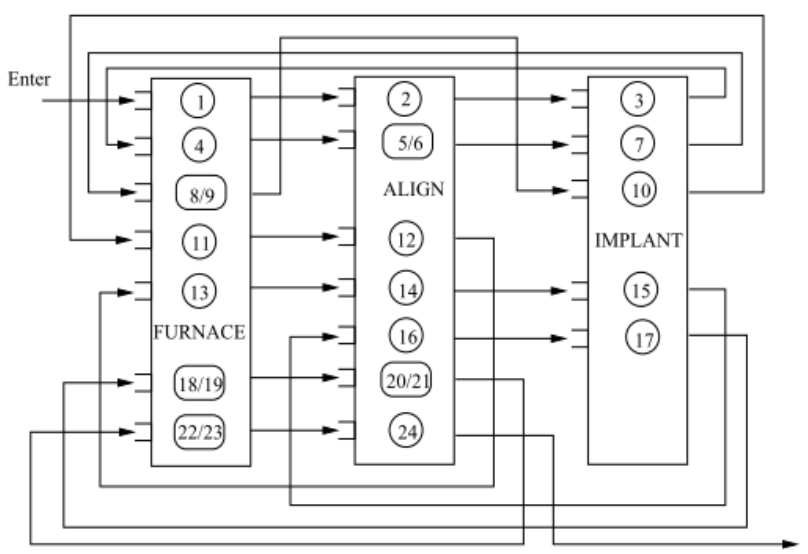

Fig2. Typical section of the production line structure [22,c.177]

$Y(t, S)$ (figure 1). The latter is due to the fact that modern production lines in the composition of the aggregated technological module contain a sufficiently large amount of equipment of different productivity that can work sequentially, in parallel or in combination (figture 2 ) $[6,7,27,28,33]$. The models of optimal control of switching on the backup equipment (identical, equipment of different productivity) to ensure the required capacity of the production line are discussed in detail in the work [34]. We introduce the function $\omega_{Y}(t, S)$, characterizing the cost of technological resources for the implementation of control $Y(t, S)$. technological resources needed to manage $Y(t, S)$ within $m-t h$ a technological operation for the duration of the production cycle $T_{d}$ determined by the integral

$$
\int_{0}^{T_{d}} \int_{S_{m-1}}^{S_{m}} Y(t, S) \cdot \omega_{Y}(t, S) d S d t \quad(\$),
$$

and the total cost of managing the state of inter-operational reserves and the performance of technological areas for all operations of the technological route by the integral of the form

$$
\int_{0}^{T_{d}} \int_{0}^{S_{d}} Y(t, S) \cdot \omega_{Y}(t, S) d S d t \quad(\$)
$$

The parameters of the production line for continuous production with a sufficiently large number of technological operations satisfy the system of balance equations [12]. For a one-step approximation, the system of balance equations takes the form:

$$
\frac{\partial[\chi]_{0}(t, S)}{\partial t}+\frac{\partial[\chi]_{1}(t, S)}{\partial S}=0, \quad[\chi]_{1}(t, S)=[\chi]_{1 \psi}(t, S) \text {. }
$$

The normative rate $[\chi]_{1 \psi}(t, S)$ of processing items of labor for the production line is set at each point of the technological route and for each point in time. As indicated above, the flow parameters in the model for managing the parameters of the production line are interoperative backlogs, which characterize the density $[\chi]_{0}(t, S)$ of distribution of technological objects of labor along the technological route $[\chi]_{1}(t, S)$ along the route [35-37]. The closedness of the balance system of equations for the parameters of the flow description level (macro level) in the continuity equation is provided using the equations of the subject-technological description level (micro level) [38].

Behavior of stream parameters $[\chi]_{0}(t, S),[\chi]_{1}(t, S)$ production line constrained by the initial and final conditions of the distribution of objects of labor on the technological route

$$
[\chi]_{0}(0, S)=[\chi]_{00}(S), \quad[\chi]_{0}\left(T_{d}, S\right)=[\chi]_{0 T_{d}}(S)
$$

the boundary conditions determining the receipt from the warehouse of raw materials, materials for the first technological operation and the output of the finished product from the last technological operation: 


$$
[\chi]_{0}(t, 0)=[\chi]_{0 S}(t), \quad[\chi]_{1}(t, 0)=[\chi]_{1 S}(t)
$$

storage capacity restrictions

$$
[\chi]_{0 G}(S) \geq[\chi]_{0}(t, S) \geq 0 \text {. }
$$

Strict inequality corresponds to the continuous mode of operation of the production line

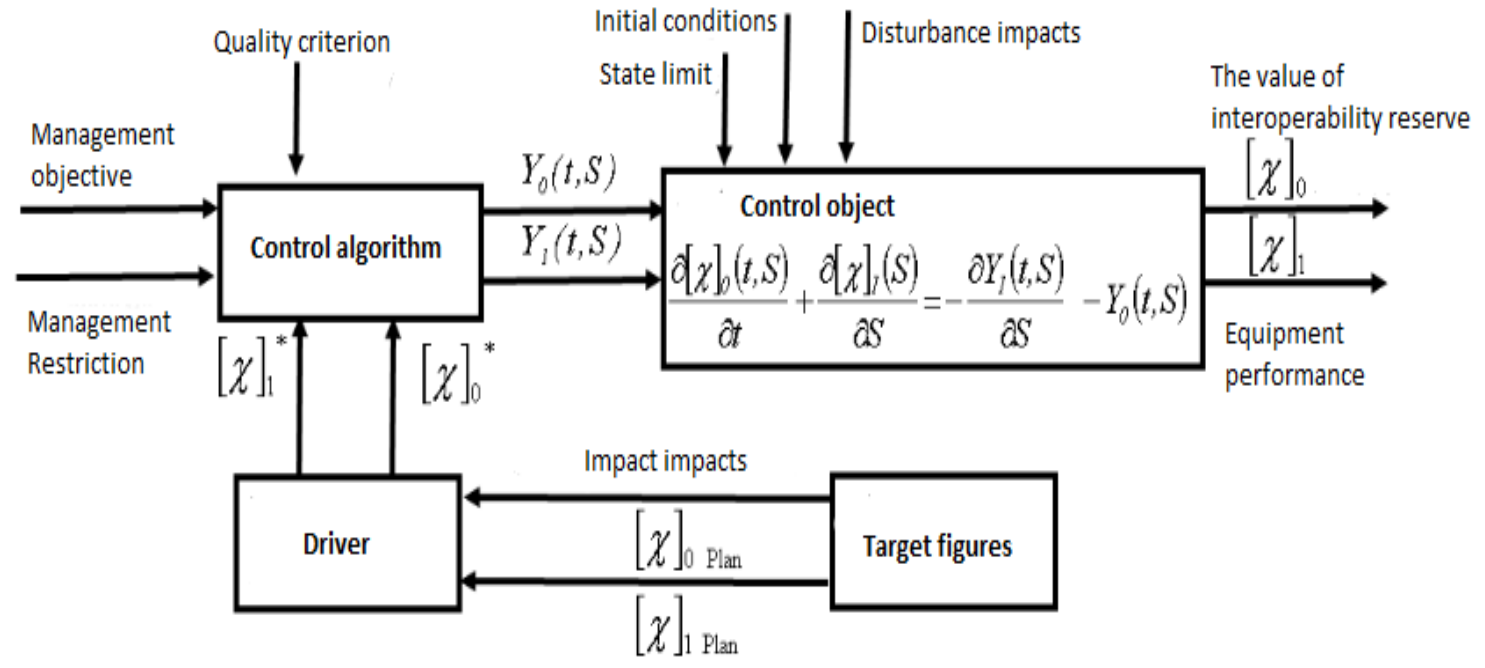

Fig3. Block diagram of the software control parameters of the production line

\section{Mathematical Formulation of the Program Control Problem}

Mathematical formulation of the program control problem. In general, the task of building an optimal program $[12,14,39]$ for managing interoperability $[\chi]_{0}(t, S)$ production line using to achieve the objectives of the control of additional equipment can be formulated as follows: to determine the status of interoperability backlog $[\chi]_{0}(t, S) \in G_{0}$ production line for each point $S \in\left[0, S_{d}\right]$ technological route for a period of time $t \in\left[0, T_{d}\right]$ while managing the performance of process equipment $Y(t, S) \in G_{Y}$ on $\mathrm{m}$ technological operations delivering a minimum of functionality [14, p.17]

$\int_{0}^{T_{d}} \int_{0}^{S_{d}}\left(Y_{1}(t, S) \cdot \omega_{Y 1}(t, S)+Y_{0}(t, S) \cdot \omega_{Y 0}(t, S)\right) d S d t \rightarrow \min (\$)$,

with differential connections

$$
\frac{\partial[\chi]_{0}(t, S)}{\partial t}+\frac{\partial[\chi]_{1}(S)}{\partial S}=-\frac{\partial Y_{1}(t, S)}{\partial S}-Y_{0}(t, S), \quad[\chi]_{1}(t, S)=[\chi]_{1 \psi}(t, S),
$$

which are determined by the system of balance equations of the two-level model of the controlledproduction process, the restrictions along the trajectory on the phase variables $[\chi]_{0}(t, S)_{[14 \text {, }}$ p.21], determined by drive capacity [40]:

$0 \leq[\chi]_{0}(t, S),[\chi]_{0}(t, S) \leq[\chi]_{0 G}(S)$,

constraints along the control path [14, p.20]

$0 \leq Y(t, S), \quad Y(t, S)+[\chi]_{1}(t, S) \leq[\chi]_{1 G}, \quad Y(t, S)=\int_{0}^{S} Y_{0}(t, \zeta) d \zeta+Y_{1}(t, S)$,

initial conditions

$$
[\chi]_{0}(0, S)=[\chi]_{00}(S) \text {, }
$$

final state (control goal)

$$
[\chi]_{0}\left(T_{d}, S\right)=[\chi]_{0 T_{d}}(S)
$$


and boundary conditions

$$
[\chi]_{1}(t, 0)=[\chi]_{1 \psi}(0), \quad[\chi]_{1}\left(t, S_{d}\right)=[\chi]_{1 \psi}\left(S_{d}\right),
$$

Under control $Y(t, S)=\int_{0}^{S} Y_{0}(t, \zeta) \mathrm{d} \zeta+Y_{1}(t, S)$ refers to the magnitude of the processing rate of items of labor on the additionally included equipment at the location of the technological route with the coordinate $S \in\left[0, S_{d}\right]$ at the moment of time t.

Limit value $[\chi]_{1 G} \quad S$ в момент времени t. determines the maximum permissible rate of processing of items of labor in the area of the technological route with the coordinate

$$
\omega_{Y 0}(t, S)=\omega_{00} \cdot Y_{0}(t, S), \omega_{Y 1}(t, S)=\omega_{01} \cdot Y_{1}(t, S), \omega_{00}=\text { const }, \omega_{01}=\text { const } .
$$

In general $\omega_{0 \mathrm{j}}$ it can be specified as a function of time t. Dependence (14) corresponds to the production systems of the flow type of production with mass and serial production, determined by linear production functions [41], connecting the costs of technological resources $\omega_{Y}(t, S)$ with production rate $Y(t, S)$. Function $Y(t, S)$ with restrictions on phase variables (9)

and control constraints (10) ensuring the achievement of the control goal (12) with the minimum value of the integral (7) and differential constraints (8), is the optimal program or optimal contr [15,p.16] for stream parameters $[\chi]_{0}(t, S) \in G_{0}$. production line. In designing the control system, differential equation equations (8) are used, which are determined by the balance equation system of a two-level model of a controlled production process.

\section{Calculation of the Optimal Program for Managing the State of the Inter- Operational BaCklogs of THE Production Line}

To determine the optimal program of control of interoperative reserves of the production line $Y(t, S)$, $[\chi]_{0}(t, S),[\chi]_{1}(S)$ in a row Fourier on the gap $S \in\left[0, S_{d}\right]$ :

$$
\begin{aligned}
& Y_{0}(t, S)=\mathrm{Y}_{00}(t), Y_{1}(t, S)=\sum_{j=1}^{\infty}\{Y\}_{j} \cdot \sin \left[k_{j} \cdot S\right]+\sum_{j=1}^{\infty}[Y]_{j} \cdot \cos \left[k_{j} \cdot S\right], \quad k_{j}=\frac{2 \cdot \pi \cdot j}{S_{d}}, j=1 . . \infty . \\
& \{Y\}_{0}=\frac{1}{S_{d}} \int_{0}^{S_{d}} Y(t, S) d S,\{Y\}_{j}=\frac{2}{S_{d}} \int_{0}^{S_{d}} Y(t, S) \sin \left[k_{j} S\right] d S,[Y]_{j}=\frac{2}{S_{d}} \int_{0}^{S_{d}} Y(t, S) \cos \left[k_{j} S\right] d S, \\
& {[\chi]_{0}(t, S)=\left\{\chi_{0}\right\}_{0}+\sum_{j=1}^{\infty}\left\{\chi_{0}\right\}_{j} \cdot \sin \left[k_{j} \cdot S\right]+\sum_{j=1}^{\infty}\left[\chi_{0}\right]_{j} \cdot \cos \left[k_{j} \cdot S\right],} \\
& {[\chi]_{1}(t, S)=\left\{\chi_{1}\right\}_{0}+\sum_{j=1}^{\infty}\left\{\chi_{1}\right\}_{j} \cdot \sin \left[k_{j} \cdot S\right]+\sum_{j=1}^{\infty}\left[\chi_{1}\right]_{j} \cdot \cos \left[k_{j} \cdot S\right],}
\end{aligned}
$$

with decomposition coefficients $\{Y\}_{0},\{Y\}_{j},[Y]_{j},\left\{\chi_{0}\right\}_{0},\left\{\chi_{0}\right\}_{j},\left[\chi_{0}\right]_{j},\left\{\chi_{1}\right\}_{0},\left\{\chi_{1}\right\}_{j},\left[\chi_{1}\right]_{j}$.

Decomposition coefficients $\left\{\chi_{1}\right\}_{0},\left\{\chi_{1}\right\}_{j},\left[\chi_{1}\right]_{j}$ will be considered known and independent of time, $\{Y\}_{0},\{Y\}_{j},[Y]_{j},\left\{\chi_{0}\right\}_{0},\left\{\chi_{0}\right\}_{j},\left[\chi_{0}\right]_{j}$ to be determined [42,p.356]. The sequences (15) - (17) are orthonormal systems of functions [43], whose coefficients can be found using the Euler - Fourier formulas [42,p.357]. The choice of the corresponding orthonormal system of functions is determined by the formulation of the problem and must provide the best approximation to the exact solution $[42,43]$. 
Taking into account (15) - (17), the quality criterion (4.7) can be integrated up to a constant in the form $[44$, p.46]

$$
\int_{0}^{T_{d}}\left(\omega_{00} \cdot Y_{00}{ }^{2}+\frac{\omega_{01}}{2} \sum_{j=1}^{\infty}\{Y\}_{j}^{2}+\frac{\omega_{01}}{2} \sum_{j=1}^{\infty}[Y]_{j}^{2}\right) d t \rightarrow \min
$$

The constraints along the trajectory on the phase variables and the control, as well as the initial, boundary conditions and the control target, taking into account (15) - (17), can be written as follows:condition of non-negativity

$$
\left\{\chi_{0}\right\}_{0}=0, \quad\left\{\chi_{0}\right\}_{j}=0, \quad\left[\chi_{0}\right]_{j}=0 \quad \text { at } 0>[\chi]_{0}(t, S)
$$

- limited storage capacity for interoperable stocks

$\left\{\chi_{0}\right\}_{0}=\left\{\chi_{0 G}\right\}_{0},\left\{\chi_{0}\right\}_{j}=\left\{\chi_{0 G}\right\}_{j}\left[\chi_{0}\right]_{j}=\left[\chi_{0 G}\right]_{j}$, at $[\chi]_{0}(t, S)>[\chi]_{0 G}(S)$

- control constraint

$\{Y\}_{0}=0 ; \quad\{Y\}_{j}=0 ; \quad[Y]_{j}=0 ; \quad$ at $\quad 0>Y(t, S)$

$0=\left\{\chi_{1 G}\right\}_{0}-\left\{\chi_{1}\right\}_{0}-\mathrm{Y}_{00} \frac{S_{d}}{2},\{Y\}_{j}=\left\{\chi_{1 G}\right\}_{j}-\left\{\chi_{1}\right\}_{j}+\mathrm{Y}_{00} \frac{2}{k_{j}},[Y]_{j}=\left[\chi_{1 G}\right]_{j}-\left[\chi_{1}\right]_{j}$,

at $Y(t, S) \geq[\chi]_{1 G}-[\chi]_{1}(t, S)$

- under initial conditions

$$
\left.\left\{\chi_{0}\right\}_{0}\right|_{t=0}=\left\{\chi_{00}\right\}_{0},\left.\quad\left\{\chi_{0}\right\}_{j}\right|_{t=0}=\left\{\chi_{00}\right\}_{j},\left.\quad\left[\chi_{0}\right]_{j}\right|_{t=0}=\left[\chi_{00}\right]_{j},
$$

and control objectives

$$
\left.\left\{\chi_{0}\right\}_{0}\right|_{t=T_{d}}=\left\{\chi_{0 T_{d}}\right\}_{0},\left.\quad\left\{\chi_{0}\right\}_{j}\right|_{t=T_{d}}=\left\{\chi_{0 T_{d}}\right\}_{j},\left.\quad\left[\chi_{0}\right]_{j}\right|_{t=T_{d}}=\left[\chi_{0 T_{d}}\right]_{j} .
$$

and specified regulatory parameters of the technological equipment along the technological route $\left\{\chi_{1}\right\}_{0},\left\{\chi_{1}\right\}_{j},\left[\chi_{1}\right]_{j}$. Taking into account the form of the target functional (18) with differential constraints

$$
\frac{d\left\{\chi_{0}\right\}_{0}}{d t}=-\mathrm{Y}_{00}, \quad \begin{cases}\frac{d\left\{\chi_{0}\right\}_{j}}{d t}-k_{j} \cdot\left[\chi_{1}\right]_{j}=k_{j} \cdot[Y]_{j} ; & k_{j}=\frac{2 \cdot \pi \cdot j}{S_{d}}, \\ \frac{d\left[\chi_{0}\right]_{j}}{d t}+k_{j} \cdot\left\{\chi_{1}\right\}_{j}=-k_{j} \cdot\{Y\}_{j} ; & j=1 . . \infty .\end{cases}
$$

which are determined by the system of balance equations (8), we write the Pontryagin function for the system under study

$$
H=-\psi_{0} \mathrm{Y}_{00}-\omega_{00} \mathrm{Y}_{00}{ }^{2}+\sum_{j=1}^{\infty}\left(\psi_{s j} k_{j}\left([Y]_{j}+\left[\chi_{1}\right]_{j}\right)-\psi_{c j} k_{j}\left(\{Y\}_{j}+\left\{\chi_{1}\right\}_{j}\right)-\frac{\omega_{01}}{2}\{Y\}_{j}{ }^{2}-\frac{\omega_{01}}{2}[Y]_{j}\right)
$$

Taking into account the restrictions (19) - (22) on the phase coordinates, the Lagrangian of the system under study takes the form

$$
\begin{aligned}
& L=-\psi_{0} \gamma_{00}-\omega_{00} r_{00}^{2}+\sum_{j=1}^{\infty}\left(\psi_{s j} k_{j}\left([Y]_{j}+\left[\chi_{1}\right]_{j}\right)-\psi_{c j} k_{j}\left(\{Y\}_{j}+\left\{\chi_{1}\right\}_{j}\right)-\frac{\omega_{01}}{2}\{Y\}_{j}^{2}-\frac{\omega_{01}}{2}[Y]_{j}\right)+ \\
& +\sum_{j=1}^{\infty}\left(\mu_{s j}\left\{\chi_{0}\right\}_{j}+\mu_{c j}\left[\chi_{0}\right]_{j}\right)+\mu_{0}\left\{\chi_{0}\right\}_{0}+\sum_{j=1}^{\infty}\left(\lambda_{s j}\left(\left\{\chi_{0 G}\right\}_{j}-\left\{\chi_{0}\right\}_{j}\right)+\lambda_{c j}\left(\left[\chi_{0 G}\right]_{j}-\left[\chi_{0}\right]_{j}\right)\right)+\lambda_{0}\left(\left\{\chi_{0 G}\right\}_{0}-\left\{\chi_{0}\right\}_{0}\right)
\end{aligned}
$$

where 
$\mu_{0} \cdot\left\{\chi_{0}\right\}_{0}=0, \quad \mu_{0} \geq 0 ; \quad \mu_{s j} \cdot\left\{\chi_{0}\right\}_{j}=0, \quad \mu_{s j} \geq 0 ; \quad \mu_{c j} \cdot\left[\chi_{0}\right]_{j}, \quad \mu_{c j} \geq 0 ;$

$\lambda_{0} \cdot\left(\left\{\chi_{0 G}\right\}_{0}-\left\{\chi_{0}\right\}_{0}\right)=0, \lambda_{0} \geq 0 ; \lambda_{s j} \cdot\left(\left\{\chi_{0 G}\right\}_{j}-\left\{\chi_{0}\right\}_{j}\right)=0, \lambda_{s j} \geq 0 ; \lambda_{c j} \cdot\left(\left[\chi_{0 G}\right]_{j}-\left[\chi_{0}\right]_{j}\right)=0, \lambda_{c j} \geq 0$

The conjugate system for Lagrangian has the form

$\frac{d \psi_{0}}{d t}=-\left(\mu_{0}-\lambda_{0}\right) ; \quad \frac{d \psi_{s j}}{d t}=-\left(\mu_{s j}-\lambda_{s j}\right) ; \quad \frac{d \psi_{c j}}{d t}=-\left(\mu_{c j}-\lambda_{c j}\right)$.

The control at which the maximum of the control function is reached is determined from the system of equations

$\frac{d L}{d \mathrm{Y}_{00}}=-\psi_{0}-2 \omega_{00} \cdot \mathrm{Y}_{00}=0, \frac{d L}{d\{Y\}_{j}}=-\psi_{c j} \cdot k_{j}-\omega_{01}\{Y\}_{j}=0, \frac{d L}{d[Y]_{j}}=\psi_{s j} \cdot k_{j}-\omega_{01}[Y]_{j}=0$,

whence the expansion coefficients are determined from the equation

$\mathrm{Y}_{00}=-\frac{\psi_{0}}{2 \omega_{00}}, \quad\{Y\}_{j}=-\frac{\psi_{c j} \cdot k_{j}}{\omega_{01}}, \quad[Y]_{j}=\frac{\psi_{s j} \cdot k_{j}}{\omega_{01}}$,

if the control value is within the control change interval and looks like (21) or (22), if it is outside of it. We substitute (32) into (25), we obtain the system of equations for determining the phase trajectory and control

$\frac{d\left\{\chi_{0}\right\}_{0}}{d t}=\frac{\psi_{0}}{2 \omega_{00}}, \quad \begin{cases}\frac{d\left\{\chi_{0}\right\}_{j}}{d t}=\frac{\psi_{s j} \cdot k_{j}^{2}}{\omega_{01}}+k_{j} \cdot\left[\chi_{1}\right]_{j} ; & k_{j}=\frac{2 \cdot \pi \cdot j}{S_{d}}, \\ \frac{d\left[\chi_{0}\right]_{j}}{d t}=\frac{\psi_{c j} \cdot k_{j}^{2}}{\omega_{01}}-k_{j} \cdot\left\{\chi_{1}\right\}_{j} & j=1 . . \infty .\end{cases}$

$\psi_{0}=-\left(\mu_{0}-\lambda_{0}\right) \cdot t+\psi_{00} ; \quad \psi_{s j}=-\left(\mu_{s j}-\lambda_{s j}\right) \cdot t+\psi_{s j 0} ; \quad \psi_{c j}=-\left(\mu_{c j}-\lambda_{c j}\right) \cdot t+\psi_{c j 0}$.

From the terms of additional slackness (28), (29) at $0<[\chi]_{0}(t, S),[\chi]_{0}(t, S)<[\chi]_{0 G}(S)$ solution (33) has the form:

$$
\left\{\chi_{0}\right\}_{0}=\frac{\psi_{0}}{2 \omega_{00}} \cdot t+\left\{\chi_{0}\right\}_{00} ; \quad\left\{\begin{array}{l}
\left\{\chi_{0}\right\}_{j}=\frac{\psi_{s j 0} \cdot k_{j}^{2}}{\omega_{01}} \cdot t+k_{j} \cdot\left[\chi_{1}\right]_{j} \cdot t+\left\{\chi_{0}\right\}_{j 0} ; \\
{\left[\chi_{0}\right]_{j}=\frac{\psi_{c j 0} \cdot k_{j}^{2}}{\omega_{01}} \cdot t-k_{j} \cdot\left\{\chi_{1}\right\}_{j} \cdot t+\left[\chi_{0}\right]_{j 0} .}
\end{array}\right.
$$

Conjugate functions can change the sign once on the path corresponding to the constraint. Therefore, the switch point for (32) is outside the control interval. Define the integration constant $\left\{\chi_{0}\right\}_{00},\left\{\chi_{0}\right\}_{j 0}$ and $\left[\chi_{0}\right]_{j 0}$ from the initial condition (23) and control objectives (24)

$$
\begin{gathered}
\left\{\chi_{0}\right\}_{0}(0)=\left\{\chi_{0}\right\}_{00}=\left\{\chi_{00}\right\}_{0} ; \\
\left\{\chi_{0}\right\}_{0}\left(T_{d}\right)=\frac{\psi_{0}}{2 \omega_{00}} T_{d}+\left\{\chi_{0}\right\}_{00}=\left\{\chi_{0 T_{d}}\right\}_{0} ;\left\{\begin{array}{l}
\left\{\begin{array}{l}
\left\{\chi_{0}\right\}_{j}(0)=\left\{\chi_{0}\right\}_{j 0}=\left\{\chi_{00}\right\}_{j} ; \\
\left.\left[\chi_{0}\right]_{j}(0)=\left[\chi_{d}\right)=\frac{\psi_{s j 0} k_{j 0}{ }^{2}}{\omega_{01}} T_{d}+\chi_{00}\right]_{j} .
\end{array}\right. \\
{\left[\chi_{0}\right]_{j}\left(\chi_{d}\right)=\frac{\left.\psi_{c j 0}\right]_{j} T_{j} T_{d}+\left\{\chi_{0}\right\}_{j 0}=\left\{\chi_{0 T_{d}}\right\}_{j} ;}{\omega_{01}} T_{d}-k_{j}\left\{\chi_{1}\right\}_{j} T_{d}+\left[\chi_{0}\right]_{j 0}=\left[\chi_{0 T_{d}}\right]_{j} .}
\end{array}\right.
\end{gathered}
$$

Allow system (36), (37) with respect to adjoint functions:

$$
\psi_{0}=2 \omega_{00} \frac{\left\{\chi_{0 T_{d}}\right\}_{0}-\left\{\chi_{0}\right\}_{00}}{T_{d}},\left\{\begin{array}{l}
\psi_{s j 0}=\frac{\omega_{01}}{k_{j}{ }^{2}}\left(\frac{\left\{\chi_{0 T_{d}}\right\}_{j}-\left\{\chi_{0}\right\}_{j 0}}{T_{d}}-k_{j} \cdot\left[\chi_{1}\right]_{j}\right), \\
\psi_{c j 0}=\frac{\omega_{01}}{k_{j}{ }^{2}}\left(\frac{\left[\chi_{0 T_{d}}\right]_{j}-\left[\chi_{0}\right]_{j 0}}{T_{d}}+k_{j} \cdot\left\{\chi_{1}\right\}_{j}\right),
\end{array}\right.
$$


taking into account (32), we obtain the expressions for the expansion coefficients (15) of the program control function of the flow parameters of the production line

$\mathrm{Y}_{00}=-\frac{\left\{\chi_{0 T_{d}}\right\}_{0}-\left\{\chi_{0}\right\}_{00}}{T_{d}}, \quad\{Y\}_{j}=-\frac{\left\lfloor\chi_{0 T_{d}}\right\rfloor_{j}-\left[\chi_{0}\right]_{j 0}}{k_{j} \cdot T_{d}}-\left\{\chi_{1}\right\}_{j}, \quad[Y]_{j}=\frac{\left\{\chi_{0 T_{d}}\right\}_{j}-\left\{\chi_{0}\right\}_{j 0}}{k_{j} \cdot T_{d}}-\left[\chi_{1}\right]_{j}$.

We construct a software control for the production line with a given processing rate and initial distribution of objects of labor along the technological route.

Using the expressions for the decomposition coefficients (39) and neglecting the edge effects when stopping the production line, assuming that the data existence time is much less than the stopping time, we obtain the decomposition coefficients for controlling the process of stopping the production line. The calculation of the optimal program for controlling the parameters of the production line for the modified PDE-model M / M / 1 queue [45]. The initial distribution of inter-operational reserves along the technological route is set $S_{d}=2 \pi$

$[\chi]_{0}(0, S)=\left\{\chi_{0 I n p}\right\}_{0}+\left\{\chi_{0 I n p}\right\}_{1} \sin \left[\frac{2 \pi S}{S_{d}}\right], \quad\left\{\chi_{0 I n p}\right\}_{0}=10000(\mathrm{pcs} / \$), \quad\left\{\chi_{0 I n p}\right\}_{1}=2000(\mathrm{pcs} / \$$.

for a production line with technological equipment, the performance of which, depending on the position, is determined by the expression

$[\chi]_{1}(t, S)=\left\{\chi_{1}\right\}_{0}+\left[\chi_{1}\right]_{1} \cos \left[\frac{2 \pi S}{S_{d}}\right],\left\{\chi_{1}\right\}_{0}=100$ (pcs/hour), $\left\{\chi_{1}\right\}_{1}=-50$ (pcs/hour).

It is necessary for time $T_{d}=100$ (hour) to reduce the number of interoperable reserves by moving from the initial distribution of interoperative backlogs to a given final distribution.

$$
\begin{aligned}
{[\chi]_{0}\left(T_{d}, S\right) } & =\left\{\chi_{0 O u t}\right\}_{0}+\left[\chi_{00 u t}\right]_{1} \sin \left[\frac{2 \pi S}{S_{d}}\right],\left\{\chi_{0 O u t}\right\}_{0}=9000(\mathrm{pcs} / \$ .), \\
{\left[\chi_{0 O u t}\right]_{1} } & =-1000(\mathrm{pcs} / \$) .
\end{aligned}
$$

The value $S_{d}=2 \pi$ is used to simplify calculations..

For a description of the behavior of production line parameters for a controlled production process, we use a modified PDE model M/M/1 queues [45]:

$$
\frac{\partial[\chi]_{0}(t, S)}{\partial t}+\frac{\partial F(t, S)}{\partial S}=0, \quad F(t, S)=[\chi]_{0}(t, S) v(t, S), v(t, S)=\frac{\mu_{\text {out }}}{[\chi]_{0}(t, S)+\frac{M}{\Delta S_{\psi}(t, S)}},
$$

where - the number of objects of labor that are in technological processing (the number of units of technological equipment, assuming that processing of one product is allowed on one unit of equipment), $\Delta S_{\psi}(t, S)$ - the length of the area within the execution of the technological operation, $\mu_{\text {out }}$ - the rate of movement of the object of labor at the exit from the production line.

Whereas for the production line under consideration $[\chi]_{0}(t, S) \Delta S_{\psi}>M$, equation (40) takes the form

$$
\frac{\partial[\chi]_{0}(t, S)}{\partial t}+\frac{\partial \mu_{\text {out }}}{\partial S} \approx 0, \quad v(t, S) \approx \frac{\mu_{\text {out }}}{[\chi]_{0}(t, S)} .
$$

For a given transient mode, we formulate the program control problem. Define the state of interoperability $[\chi]_{0}(t, S) \in G_{0}$ production line for each point $S \in\left[0, S_{d}\right]$ technological route for a period of time $t \in\left[0, T_{d}\right]$ while managing the performance of process equipment $Y(t, S) \in G_{Y}$ on m technological operation that delivers a minimum of functionality [15] 
$\frac{1}{S_{d}} \int_{0}^{T_{d}} \int_{0}^{S_{d}}\left(Y_{1}(t, S)+\alpha_{d} \cdot Y_{0}(t)\right)^{2} d S d t \rightarrow \min$,

with differential connections

$\frac{\partial[\chi]_{0}(t, S)}{\partial t}=-\frac{\partial Y_{1}(t, S)}{\partial S}-Y_{0}(t)$

constraints along the trajectory of the phase variables $[\chi]_{0}(t, S)[15, \mathrm{c} .21]$, determined by drive capacity

$0 \leq[\chi]_{0}(t, S),[\chi]_{0}(t, S) \leq[\chi]_{0 G}(S)$,

constraints along the control path $[14, \mathrm{p} .20]$ :

$0 \leq Y(t, S), \quad Y(t, S)+[\chi]_{1}(t, S) \leq[\chi]_{1 G}, \quad Y(t, S)=Y_{0}(t) \cdot S+Y_{1}(t, S)$,

initial conditions $[\chi]_{0}(0, S)$, control objectives $[\chi]_{0}\left(T_{d}, S\right)$ and boundary conditions

$$
[\chi]_{1}(t, 0)=[\chi]_{1 \psi}(0)=\lambda_{\text {inp }} ; \quad[\chi]_{1}\left(t, S_{d}\right)=[\chi]_{1 \psi}\left(S_{d}\right)=\mu_{\text {out }},
$$

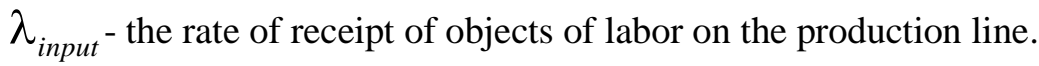

To determine the program of control of interoperative reserves of the production production line of the function $[\chi]_{0}(t, S), Y_{1}(t, S)$ present in the form:

$$
Y_{1}(t, S)=\{Y\}_{1} \sin \left[\frac{2 \pi S}{S_{d}}\right]+[Y]_{1} \cos \left[\frac{2 \pi S}{S_{d}}\right], \quad[\chi]_{0}(t, S)=\left\{\chi_{0}\right\}_{0}+\left\{\chi_{0}\right\}_{1} \sin \left[\frac{2 \pi S}{S_{d}}\right]+\left[\chi_{0}\right]_{1} \cos \left[\frac{2 \pi S}{S_{d}}\right] .
$$

Taking this into account, the transition quality criterion (42) takes the following form

$$
\int_{0}^{T_{d}}\left(\alpha_{d}^{2} \cdot Y_{0}^{2}+\frac{\{Y\}_{1}^{2}}{2}+\frac{[Y]_{1}^{2}}{2}\right) d t \rightarrow \min
$$

with equations of differential relations defined by the balance equation of the model of a controlled production process $(41)$

$$
\frac{d\left\{\chi_{0}\right\}_{0}}{d t}=-\mathrm{Y}_{0} ; \quad \frac{d\left\{\chi_{0}\right\}_{1}}{d t}=\frac{2 \pi}{S_{d}}[Y]_{1} ; \frac{d\left[\chi_{0}\right]_{1}}{d t}=-\frac{2 \pi}{S_{d}}\{Y\}_{1} .
$$

We write the function Pontryagin, which allows to determine the program for controlling the parameters of the production line of the production and technical system

$H=-\alpha_{d}^{2} \cdot Y_{0}^{2}-\frac{\{Y\}_{1}^{2}}{2}-\frac{[Y]_{1}^{2}}{2}-\psi_{0} \cdot Y_{0}+\psi_{s} \cdot \frac{2 \pi}{s_{d}} \cdot[Y]_{1}-\psi_{c} \cdot \frac{2 \pi}{S_{d}} \cdot\{Y\}_{1}$.

The conjugate system can be obtained as:

$\frac{d \psi_{0}}{d t}=0 ; \frac{d \psi_{s}}{d t}=0 ; \frac{d \psi_{c}}{d t}=0$,

which makes it possible to determine the expansion coefficients for optimal control from the system of equations

$\frac{d H}{d \Upsilon_{0}}=-\psi_{0}-2 \alpha_{d}^{2} \Upsilon_{0}=0, \frac{d H}{d\{Y\}_{1}}=-\psi_{c} \frac{2 \pi}{S_{d}}-\{Y\}_{1}=0, \frac{d H}{d[Y]_{1}}=\psi_{s} \frac{2 \pi}{S_{d}}-[Y]_{1}=0$,

from where

$$
\mathrm{Y}_{0}=-\frac{\psi_{0}}{2 \alpha_{d}^{2}}, \quad\{Y\}_{1}=-\psi_{c} \frac{2 \pi}{S_{d}},[Y]_{1}=\psi_{s} \frac{2 \pi}{S_{d}},
$$


if its value is within the control change interval and is determined from the inequalities that determine the control constraints, if it is outside its limits. Substitute the obtained expressions into the equations of differential constraints, we obtain a system of equations for determining the phase trajectory:

$\frac{d\left\{\chi_{0}\right\}_{0}}{d t}=\frac{\psi_{0}}{2 \alpha_{d}^{2}} ; \quad \frac{d\left\{\chi_{0}\right\}_{1}}{d t}=\left(\frac{2 \pi}{S_{d}}\right)^{2} \psi_{s} ; \quad \frac{d\left[\chi_{0}\right]_{1}}{d t}=\left(\frac{2 \pi}{S_{d}}\right)^{2} \psi_{c} ;$

whose solution

$\left\{\chi_{0}\right\}_{0}=\frac{\psi_{0}}{2 \alpha_{d}^{2}} \cdot t+\left\{\chi_{0 I n p}\right\}_{0} ;\left\{\chi_{0}\right\}_{1}=\left(\frac{2 \pi}{S_{d}}\right)^{2} \psi_{s} \cdot t+\left\{\chi_{0 I n p}\right\}_{1} ;\left[\chi_{0}\right]_{j}=\left(\frac{2 \pi}{S_{d}}\right)^{2} \psi_{c} \cdot t+\left[\chi_{0 I n p}\right]_{1}$.

satisfies the initial conditions and control objectives. Allow system

$$
\left\{\chi_{0 O u t}\right\}_{0}=\frac{\psi_{0}}{2 \alpha_{d}^{2}} \cdot T_{d}+\left\{\chi_{0 I n p}\right\}_{0} ; \quad\left\{\chi_{0 O u t}\right\}_{1}=\left(\frac{2 \pi}{S_{d}}\right)^{2} \psi_{s} \cdot T_{d}+\left\{\chi_{0 I n p}\right\}_{1} ;\left[\chi_{0 O u t}\right]_{1}=\left(\frac{2 \pi}{S_{d}}\right)^{2} \psi_{c} \cdot T_{d}+\left[\chi_{0 I n p}\right]_{1} \text {. }
$$

with respect to conjugate functions $\psi_{0}, \psi_{s}, \psi_{c}$

$\psi_{0}=2 \alpha_{d}{ }^{2} \frac{\left\{\chi_{0 O u t}\right\}_{0}-\left\{\chi_{0 I n p}\right\}_{0}}{T_{d}} ; \quad \psi_{s}=\frac{\left\{\chi_{0 O u t}\right\}_{1}-\left\{\chi_{0 I n p}\right\}_{1}}{T_{d}}\left(\frac{S_{d}}{2 \pi}\right)^{2} ; \quad \psi_{c}=\frac{\left[\chi_{0 \text { Out }}\right]_{1}-\left\lfloor\chi_{0 I n p}\right]_{1}}{T_{d}}\left(\frac{S_{d}}{2 \pi}\right)^{2}$.

we obtain the expansion coefficients for the control function:

$$
\mathrm{Y}_{0}=-\frac{\left\{\chi_{0 O u t}\right\}_{0}-\left\{\chi_{0 I n p}\right\}_{0}}{T_{d}}, \quad\{Y\}_{1}=-\frac{\left\{\chi_{0 O \text { out }}\right\}_{1}-\left\{\chi_{0 I n p}\right\}_{1}}{T_{d}}\left(\frac{S_{d}}{2 \pi}\right), \quad[Y]_{1}=\frac{\left[\chi_{0 O u t}\right]_{1}-\left\lfloor\chi_{0 I n p}\right]_{1}}{T_{d}}\left(\frac{S_{d}}{2 \pi}\right) \text {. }
$$

calculated values of which after substitution of values

$$
\mathrm{Y}_{0}=10 \text { (pcs./(hour*\$)), } \quad\left\{Y_{1}=20 \text { (pcs/hour), } \quad[Y]_{1}=-10\right. \text { (pcs/hour). }
$$

\section{CONCLUSION}

It is shown that along with the traditional models of control of parameters of production flow lines, the control models associated with the use of partial differential equations (PDE models) play a significant role. The main types of models for controlling the parameters of production flow lines are defined. A PDE model for controlling the parameters of the production flow line is proposed, taking into account restrictions on the storage capacity and control value along the technological route.

A PDE model has been developed to control the parameters of the production flow line for the regimes of reducing and increasing the volume of interoperable reserves. It is shown that the optimal control for the transitional mode of operation is determined by the first members of the expansion in orthonormal systems of functions whose coefficients are found using the Euler - Fourier formulas. The choice of an orthonormal system of functions is determined by the characteristics of the flow of the production process. Edge effects associated with filling technological positions with free processing line are estimated. The main features associated with the construction of a PDE-model for controlling the parameters of an industrial production line are considered. A target function is recorded that determines the quality criterion for controlling the parameters of a production production line. It is shown that the balance equations in partial derivatives, which act as differential constraints for phase variables, are replaced by a system of equations for the coefficients of decomposition of the parameters of the production flow line, which allowed us to obtain the control function in the form of time dependence and position (coordinates) in the technological route. In determining the optimal program for controlling the parameters of the production flow line for synchronizing the performance of the equipment of the flow line, the dependence of the control function on the initial conditions is shown. The Lagrange function was obtained for programmatically controlling the parameters of the production line. The possible types of controls for different values of the adjoint functions are highlighted. It is shown that the behavior of the flow parameters of the production line for these departments is determined by the initial and boundary conditions of the distribution of objects of labor along the technological route of the production line. 


\section{REFERENCES}

[1] Berg R. Partial differential equations in modelling and control of manufacturing systems / R. Berg. Netherlands, Eindhoven Univ. Technol., 2004. - 157 p.

[2] Forrester J. Basics of enterprise cybernetics / J. Forrester. - M .: Progress, 1961. - 341 p.

[3] Pervozvansky A. A. Mathematical methods in production control / A. A. Pervozvansky. - M .: Science,1975. $-616 \mathrm{p}$.

[4] Petrov B.N. Theories of models in control processes (Information and thermodynamic aspects) / B.N. Petrov, G.M. Ulanov, I.I. Goldenblat, S.V. Ulyanov - M .: Science, 1978. - 224 p.

[5] Razumov I. M. Organization and planning of machine-building production / I. M. Razumov, L. Ya. Shecharant - Moscow: Engineering, 1974. - 592 p.

[6] Sokolitsyn S. A. Application of mathematical methods in economics and organization of machine-building production / S. A. Sokolitsyn. - L .: Engineering, 1970. - 345 p.

[7] Control of flexible production systems. Models and algorithms / E. D. Voronin and others. / Society. ed. S.V. Emelyanov. - M .: Engineering, 1987. - 368 p.

[8] V. Shkurba. Discrete production planning in the ACS / V. B. Shkurba, In .A. Boldyrev, A. A. Vyun et al. / Ed. V.M. Glushkov. - K .: Technology, 1975. - 296 p.

[9] Lefeber E. Modeling, Validation and Control of Manufacturing Systems. / E.Lefeber, R.A.Berg, J.E. Rooda — Proceeding of the 2004 American Control Conference, Massachusetts, 2004. - P. 4583 - 4588.

[10] Lefeber E. Controlling a re-entrant manufacturing line via the push-pull point. / E.Lefeber, D.Perdaen, D.Armbruster, K.Kempf- International Journal of Production Research 46(16), 2008. - P. 4521 - 4536.

[11] Mac Gregor S.J. Handbook of Stochastic Models and Analysis of Manufacturing System Operations. / S. J. Mac Gregor, B. Tan. - New York, 2013. -Vol. 192. - Series: International Series in Operations Research \& Control Science. - P. 373.

[12] Pihnastyi O. M. Statistical theory of production systems / O. M Pihnastyi. - Kharkiv: KhNU, 2007. -388 p.

[13] Krasovsky A. A. Phase space and the statistical theory of dynamical systems / A. A. Krasovsky. - M .: Science, 1974. $-232 \mathrm{p}$.

[14] Mikhailov V.S. Theory of control / V.S. Mikhailov - K: High School, 1988. - 312 p.

[15] Moiseev N. N. Elements of the theory of optimal systems / N. N. Moiseev. - M .: Science, 1974. - 526 p.

[16] Pontryagin L.S. Mathematical theory of optimal processes / L.S. Pontryagin, V.G. Boltyansky, R.V. Gamkrelidze - M .: Nauka, Main publishing house of physical and mathematical literature 1983. - $392 \mathrm{p}$.

[17] Pugachev V.S. Basics of the statistical theory of automatic systems / V.S. Pugachev, I.E. Kazakov, L.G. Evlanov. - M :: Mechanical Engineering, 1974. -400 p.

[18] Pihnistyi O. M. Calculation of the Production Cycle Using the Statistical Theory of Production-Technical Systems / O.M. Pihnastyi, V.D. Khodusov // Supplements of the National Academy of Sciences of Ukraine. - Kiev: «Academperiodika» Publishing House. - 2009. - №12. - p. 38-44.doi.org/10.13140 /RG. 2.2.36267.54562

[19] Bellman R. The theory of the stability of solutions of differential equations / R. Bellman - M .: Foreign Literature Publishing House, 1954. - 215 p.

[20] Kazakov I.Ye. Statistical theory of control systems in the state space / I.E. Cossacks. - M .: Science, 1975. $-432 \mathrm{p}$.

[21] Armbruster D. Kinetic and fluid model hierarchies for supply chains supporting policy attributes / D. Armbruster., D. Marthaler, C. Ringhofer // Bulletin of the Institute of Mathematics. - Academica Sinica, 2006. - P. $496-521$.

[22] Ramadge P. The control of discrete event systems / P. Ramadge, W. Wonham. // Proceedings of the IEEE - 1989. - vol. 77, №1. - P. 81 - 98 .

[23] Harrison J. Brownian Motion and Stochastic Flow Systems. / J. Harrison. - New York, 1995. - P. 142.

[24] Demutsky V.P. Theory of an Enterprise: Sustainability of Mass Production and Product Promotion / V.P. Demutsky, V.S. Pihnasta, O. M. Pihnastyi. - Kharkov .: KhNU, 2003. - 272 p.

[25] Armbruster D. Continuous models for production flows.. / D. Armbruster, C. Ringhofer., Jo T- J. // In Proceedings of the 2004 American Control Conference. - Boston, MA, USA. - 2004. - P. 4589 - 4594.

[26] Zaruba V. Ya. Entropy of the technological process / V. Ya. Zaruba, O.M. Pihnastyi // Control of large systems: works of the international scientific-practical conference "Theory of active systems" (TAC2011), (Moscow, November 14-16, 2011). - Moscow IMP RAS. - 2011. - vol. 2. - P. 145 - 148. 
[27] Serebrennikov G. G. Organization of production / G. G. Serebrennikov. - Tambov: Edition. TSTU, 2004. $96 \mathrm{p}$.

[28] Sinitsa L. M. Organization of production / L. M. Sinitsa. - M.: 2003. - 512 p.

[29] Tian F. An iterative approach to item-level tactical production and inventory planning. / F.Tian, S.P.Willems, K.G.Kempf -International Journal of Production Economics, 2011. - vol. 133. - P. 439 - 450

[30] Lyapilin I. I. Introduction to the theory of kinetic equations / I. I. Lyapilin. - Yekaterinburg:2003. - 205 p.

[31] Pihnisty O. M. Use of PDE - models for Building a Unified Theory of Production Lines / O.M. Pihnastyi, // The Kherson National Library Technique. Kherson: KhNTU. - 2014. - № 3 (50).- p. 405 - 412.

[32] Pihnastyi O. M. On the construction of the target function of the production system / O. M. Pihnastyi // Supplements of the National Academy of Sciences of Ukraine. - Kyiv: Publishing House "Academperiodika".- 2007. - №5. - p. 50 - 55.

[33] Feldbaum A. A. Methods of the theory of automatic control: a scientific publication / A. A. Feldbaum, A. G. Butkovsky. - M .: Science, 1971. - 744 p.

[34] Lysenko Yu. G. Modeling technological flexibility of production and economic systems / Yu. G. Lysenko, N. V. Rumyantsev. - Donetsk: DonNU, 2007. - 238 p.

[35] Pihnastyi O. M. Multi-stream model of the production line / O. M. Pihnastyi // Math model. Dniprodzerzhynsk: DDTU. - 2017. - № 1. - p. 81-87. - https://doi.org/10.13140/RG. 2.2.19038. 13128

[36] Pihnistyi O. M. The problem of optimal operative control of macroparameters of a production system with mass production. M. Pihnastyi // Dopovidi National Academy of Ukraine. - Kiev: Publishing House "Academperiodika".- 2006. - №5 - p. 79 - 85.

[37] Karcheva G. Problems and prospects of development of the banking system of Ukraine: st. sciences. Ave. / NBU; Ministry of Education and Science of Ukraine. - Sumi, 2009. - vol. 26. - p. 200-206. https://goo.gl/3HcT8P

[38] Pihnistyi O. M. Review of models of controlled production processes of production lines of production systems / O.M. Pihnastyi // Scientific statements of Belgorod State University. Belgorod: BSU. - 2015. - № 34/1. p.137-152.

[39] Lurie K. A. Optimal control in problems of mathematical physics / K. A. Lurie. - M .: Science. Main editors of physical and mathematical literature1975. $-480 \mathrm{p}$.

[40] Armbruster D. A continuous model for supply chains with nit buffers / D. Armbruster, S. Goettlich , M. Herty // SIAM Journal on Applied Mathematics. - 2011. - P. 855 - 877.

[41] Kleiner G. B. Production functions: Theory, methods, application / G. B. Kleiner. - M .: Finance and Statistics, 1986. - 239 p.

[42] Valko V. I. Variation calculus and optimal control / V. I. Valko, O. V. Ermoshina, G. N. Kuvyrkin. Moscow: Moscow State Technical University named after Bauman, 2006. - 488 p.

[43] Elsgolts L.E. Differential equations and calculus of variations/L.E.Elsgolts - Moscow: Nauka, 1969.- 425 p.

[44] Lavrentiev, M. Fundamentals of the calculus of variations / M. Lavrentyev, L. Lyusternik. - M .: Publishing house NKTP USSR, 1935. - vol. 2. - 399 p.

[45] Armbruster D. Kinetic and fluid model hierarchies for supply chains. / D. Armbruster., D. Marthaler, C. Ringhofer // SIAM Multiscale Model Simul.- 2004. - vol. 2 №1. - P. 43 - 61.

Citation: Pihnastyi, O. (2019). Optimal Control of the Parameters of the Production Line. International Journal of Scientific and Innovative Mathematical Research (IJSIMR), 7(2), pp.1-12.http://dx .doi.org /10.20431/2347-3142.0702001

Copyright: (C) 2019 Authors, This is an open-access article distributed under the terms of the Creative Commons Attribution License, which permits unrestricted use, distribution, and reproduction in any medium, provided the original author and source are credited. 